\title{
Improvement of Rainfall Forecast by Assimilations of Ground-Based GPS Data and Radio Occultation Data
}

\author{
Hiromu Seko, Masaru Kunii, Yoshinori Shoji and Kazuo Saito \\ Meteorological Research Institute, Tsukuba, Japan
}

\begin{abstract}
Impacts of three kinds of GPS-derived water vapor data, i.e., precipitable water vapor (PWV), slant water vapor (SWV) and radio occultation (RO) data, were investigated using the Meso 4D-Var system of Japan Meteorological Agency (JMA) for a heavy rainfall case on 16 July 2004 . When PWV or SWV data were assimilated individually, water vapor in the rainfall region was increased and on the northern sides was decreased, and then the shape of the rainfall region became similar to the observed one. However, the reproduced rainfall amount remained smaller than the observed one. Compared with PWV, SWV made the horizontal contrast of water vapor larger. When RO data were assimilated, the low-level water vapor was increased so that the rainfall amount was largely increased. However, the rainfall region became wider than the observed one. When SWV and RO data were assimilated simultaneously, low-level water vapor in the rainfall region and on its southern side was increased, and then both shapes of rainfall region and of rainfall amount became similar to the observed ones.
\end{abstract}

\section{Introduction}

Mesoscale rainfalls are not always reproduced by numerical models (e.g., White et al. 1999). Reasons of this difficulty are yielded from (1) errors in initial conditions as well as (2) inherent limit to predictability; (3) poor model dynamics and physics (Yoden 2007). Especially, water vapor in the initial condition affects the predicted rainfall amount, so it is important to improve water vapor distribution by data assimilation. Surface meteorological data, upper sounding data and satellite-derived water vapor data, such as SSMI, have been assimilated operationally in the Mesoscale model (MSM) of JMA. In addition to these data, GPSderived PWV data (the water vapor amount along the zenith direction) have been assimilated since 28 October 2009 by the JMA Non-hydrostatic model based Variational data Assimilation system (JNoVA).

Assimilation methods of GPS-derived water vapor data, including PWV, have been developed in the Meteorological Research Institute, and their impacts on the rainfall forecasts have been investigated by using the Mesoscale 4-Dimensional Variational data assimilation system (Meso 4D-Var) of JMA. Meso $4 \mathrm{D}$-Var, which is the former system of JNoVA, is the data assimilation system developed for the operational mesoscale prediction in JMA (Koizumi et al. 2005). Seko et al. (2004) proposed an assimilation method for GPS-derived PWV and SWV (water vapor amount along slant path from GPS satellite to GPS receiver), and examined their impacts on the heavy rainfall event which occurred in Nerima, Tokyo on 21 July 1999. They showed that the assimilation of PWV or SWV reproduced the size of rainfall region though its position was shifted. They also suggested that SWV had the potential to reproduce the water vapor distribution more realistically than PWV. Besides PWV and SWV data, GPS signal delays observed by low-earth orbit (LEO) satellites provide vertical profiles of refractive index (RI), which are the function of temperature, pressure of dry air and water vapor. Seko et al.

Corresponding author: Hiromu Seko, Meteorological Research Institute, 1-1 Nagamine Tsukuba Ibaraki, 305-0052, Japan. E-mail: hseko@mri-jma. go.jp. (C)2010, the Meteorological Society of Japan.
(2009) developed an assimilation method of the path-averaged RI from LEO satellites to GPS satellites. They showed that RO data was also useful to improve the forecast of heavy rainfall. Because of usefulness of RO data and ground-based GPS data (i.e., PWV and SWV), development of a mesoscale observing network for improving NWP was suggested in Southeast Asia (Koh and Teo 2009).

Although the impacts of ground-based GPS data and RO data were investigated individually, the synergistic effect, namely, how the simultaneous assimilations of these data modify the water vapor distribution, has not been investigated, so far. In this study, the impacts of PWV, SWV and RO data on the heavy rainfall, and the difference of the analyzed fields are shown. In Section 2, assimilation methods of ground-based GPS and RO are briefly explained. In Section 3, a target of the assimilation experiment and the outline of the experiment are described. Results of the assimilation experiment are shown in Section 4. Section 5 is the summary.

\section{GPS-derived data and their observation operators}

\subsection{Ground-based GPS data (SWV and PWV)}

SWV data were obtained from slant total delays observed by the GPS Earth Observation Network System (GEONET) of the Geospatial Information Authority of Japan (GSI). The lowest elevations angle of slant paths we used was 15 degrees. The estimation method of slant total delay was the same as that of Shoji et al. (2004). In the present study, the vertically scaled hydrostatic delay in the slant total delay was assumed to be the same as that in the zenith delay. These delays are proportional to the surface pressure at GPS antenna. The conversion coefficients from the zenith wet delay to PWV, which can be approximated by a function of temperature at GPS antenna, were also used in estimation of SWV. The surface pressure and temperature at each GPS receiver were obtained by interpolation of surface data of meteorological observatories and the Automated Meteorological Data Acquisition System (AMeDAS) of JMA. In practice, the altitudes of GPS receivers were different from those of numerical models. The difference of water vapor amount due to the difference of altitude was corrected by assuming that the water vapor between GPS receivers and model surface was equal to the product of the observed water vapor density at the surface and the difference of their altitudes. We used GEONET sites of which altitudes were within $50 \mathrm{~m}$ from the model topography to reduce error caused by the aforementioned assumption. PWV was obtained from SWV data for each receiver. SWV were converted to vertically scaled values and then PWV was estimated by averaging the vertically scaled values. Although PWV and SWV were computed every 5 minutes, hourly data were used in the Meso 4D-Var data assimilation.

Figure 1a illustrates how the observation operator of SWV was produced. A ray-tracing method was used to determine the slant path from GPS receiver, and the path was divided into elements with the length of $100 \mathrm{~m}$. The top of the path was set to the top of the model domain $(20 \mathrm{hPa})$. Density and mixing ratio of water vapor at the center of each element was obtained by Cressman interpolation from the grid point values of the model. Influence radius of interpolation was set to $20 \mathrm{~km}$. SWV was calculated by integrating the products of density, mixing ratio of water vapor, and the element length. The observation operator for SWV is thus given as follows: 
$S W V_{\text {model }}=\sum_{\mathrm{i}=1}^{\mathrm{i}=\mathrm{i} \operatorname{top}(z=20 \mathrm{hPa})} \rho_{\mathrm{i}} l_{\mathrm{i}} q_{\mathrm{vi}}$

where $\rho, q_{\mathrm{v}}$, and $l$ are the density, mixing ratio of water vapor, and the element length of the slant path, respectively. The observation operator of PWV was obtained by the same method except for a path extending in the vertical direction. The observation errors of PWV and SWV were set to $2 \mathrm{~mm}$ and $3 \mathrm{~mm}$, respectively. Detailed procedures of the assimilation of PWV and SWV were explained in Seko et al. (2004).

\subsection{RO data}

As for RO data, the RI averaged along the path from the GPS satellite to the LEO satellite, CHAMP (CHAllenging Minisatellite Payload) was used as the observation data. The German Research Centre for Geosciences (GFZ), which launched the CHAMP satellite, provides the RI profiles at tangent points that are the lowest points of paths. In the estimation of tangent point data, the assumption of the spherical uniform distribution of the RI was used. Because this assumption was not always satisfied, path-averaged RI was reproduced from the tangent point data by the following equation;

$$
n_{\text {path }}=\sum_{\mathrm{tp}=\text { lowest_level }}^{10 \mathrm{~km} \text { level }} l_{\mathrm{tp}} n_{\mathrm{tp}} / \sum_{\mathrm{tp}=\text { lowes__level }}^{10 \mathrm{~km} \text { level }} l_{\mathrm{tp}}
$$

where $n_{\mathrm{tp}}$ is the RI and $l_{\mathrm{tp}}$ is the path length within the $\mathrm{tp}^{\text {th }}$ layer of atmosphere. The thickness of each layer was set to $200 \mathrm{~m}$ in this study. Because water vapor that influenced the RI is small at an altitude of $10 \mathrm{~km}$, the top altitude of the path was set to $10 \mathrm{~km}$. To reduce the computational cost, the paths were assumed to be straight lines.

Procedures to calculate the observation operator were as follows: First, RIs at grid points were produced from the outputs of the model (i.e., temperature, mixing ratio of water vapor and pressure). The path from a tangent point to both ends of the path, of which altitudes were $10 \mathrm{~km}$, was divided into elements with lengths of $10 \mathrm{~km}$ so that the tangent point was located at the center of the lowest element (Fig. 1b). RIs at the center of elements were obtained from the RIs at the model grid points by spatial interpolation. The path-averaged RI was produced by averaging the RIs of the elements along the path.

Besides the assumption of spherical uniform distribution, the large correlation of observation error was the problem that should be solved. The vertical correlation was considered by using the non-diagonal elements of the observation error matrix in this study. The observation error of RO data was estimated by the comparison of RIs between the observation and the first guess field. The covariance of the observation error multiplied by 0.1
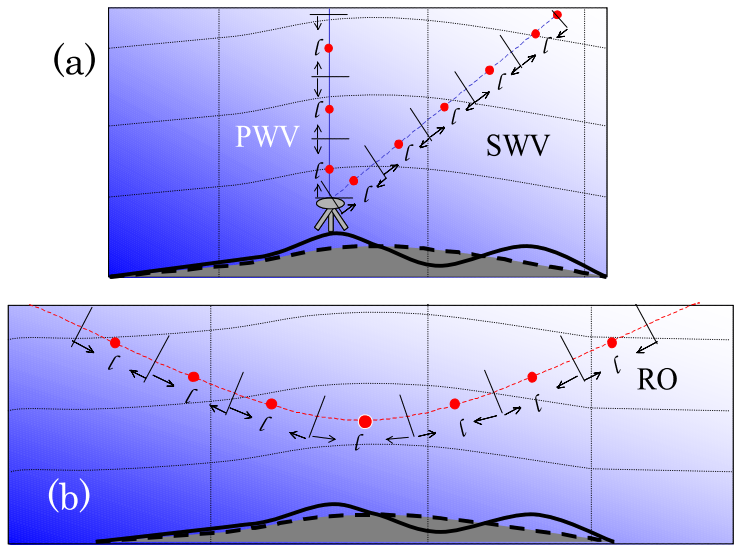

Fig. 1. Schematic illustrations of the observation operators of (a) PWV and SWV and (b) RO data. Thick solid line and broken line indicates actual topography and model topography, respectively. Thin lines indicate the planes that cross the model grids. was used to see the impact more clearly. Detailed procedures on the assimilation of RO data are explained by Seko et al. (2009).

\section{Intense rainfall event and outline of assimilation experiments}

The intense rainfall event, which is the target of the assimilation of GPS-derived data, is briefly explained. Figure $2 \mathrm{a}$ is the horizontal distribution of 3-hour rainfall amount from 12 JST to 15 JST 16 July 2004, which was estimated from the reflectivity intensity of the conventional radar and the rainfall amount of rain gauge network of JMA. On 16 July 2004, the Baiu front, which is a stationary front extending from China to Japan in early summer, stayed in northern Japan. Along this stationary front, the intense rainfall region extending from northwest to southeast was developed. At Ohyu, which existed in the intense rainfall region, a daily rainfall of $143 \mathrm{~mm}$ was recorded by AMeDAS.

As for the assimilation system in this study, Meso 4D-Var was used. The impact of the ground-based GPS data and RO data was investigated by the comparisons of the predicted rainfall intensity and the initial water vapor fields. MSM of hydrostatic version was used as the forecast model. The domains of Meso 4D-Var and MSM were the same as operational ones, which covered Japan and its surrounding areas. The horizontal grid intervals of MSM and the Meso 4D-Var analysis produced by its 'outer loop model' are $10 \mathrm{~km}$, while Meso 4D-Var adopts an incremental approach with an 'inner loop model' with a grid interval of $20 \mathrm{~km}$. The initial condition in the first assimilation window and lateral boundary conditions were supplied from the Regional Spectral Model (RSM) of JMA. Assimilation period was 09 JST to 15 JST on 16 July 2004 , just before the occurrence of the intense rainfall.

Figure $2 \mathrm{~b}$ is the 3 -hour rainfall amount predicted from the analyzed field obtained by assimilation of conventional data only, e.g., radiosonde, synop, ship, and buoy data. This experiment is hereinafter referred to as 'CNTL'. The initial time of 'CNTL' is 15 JST 16 July 2004 and the forecast time of Fig. $2 b$ is 3 hours. The rainfall amount of 'CNTL' was weaker, and the main part of the rainfall region was predicted at the eastern side of northern Japan. This result indicates that the conventional data were not enough to reproduce the intense rainfall.

\section{Results of Assimilation of PWV, SWV and RO data}

\subsection{Comparison of observed data and first guess data}

Figure 3a shows the model grids of Meso 4D-Var where the weight of spatial interpolation in observation operators of PWV, SWV and RO data was positive. Because the GPS receivers of which differences of the height from the model altitude were within $50 \mathrm{~m}$ were used, the ground GPS data over mountainous regions were removed. Although the number of data was decreased, PWV and SWV data at northern Japan still remained. These data are expected to influence the forecast of the intense rainfall. Since the SWV reaches the grids surrounding the GPS receivers, the regions of SWV data (blue dots in Fig. 3a) extend around the grids of PWV data (red dots in Fig. 3a).

Figure $3 \mathrm{~b}$ shows the differences of PWV between the observational data and first guess fields (D-value). The first guess of PWV was estimated using the observation operator that was mentioned in Section 3. Except for the region on the north of the intense rainfall, the observed PWV was larger than the first guess. Because the D-value in the intense rainfall region was positive, the rainfall amount in northern Japan is expected to be increased. Note that there were negative regions at the northern side of the intense rainfall.

As for RO data, paths of RO data passed the central Japan at 1206 JST. The tangent point of the lowest path was located at $50 \mathrm{~km}$ south of the intense rainfall region at the height of about $2 \mathrm{~km}$ (indicated by a red point in Fig. 2a). Because the paths of RO data extended to north-northeast (green dots in Fig. 3a), the northern part of the paths penetrated the intense rainfall region. 
The RIs in the path within the rainfall region are expected to be larger than those out of the rainfall region. Namely, the assumption of spherical uniform distribution was not satisfied in this event. So, the path data, namely, 'path-averaged RI' explained in Section 2.2, which does not need to satisfy the assumption, were assimilated in this study. When the RI data are used, assimilation is performed properly even over region of significant horizontal refractivity gradients, such as the region that is near the heavy rainfall.

The path-averaged RIs were produced by Eq. (2) from the tangent point data, and then compared with those of the first guess. The first guess was obtained from the output of the model using the observation operator mentioned in the previous section. When the profiles of path-averaged RI were compared, the observed values below the height of $5 \mathrm{~km}$ were larger than those of the first guess (Fig. 4). Thus, the reinforcement of the rainfall is expected by the assimilation of RO data. Because the path-averaged RIs above $6 \mathrm{~km}$ were almost the same, the top height of $10 \mathrm{~km}$ was enough in this case.

\subsection{Impact of ground-based GPS data and RO data}

Besides the experiment of 'CNTL', five experiments: 'RO', 'PWV', 'SWV', 'RO+PWV' and 'RO+SWV' were performed. In the experiments of 'PWV', 'SWV' and 'RO', PWV and SWV data from 12 JST to 15 JST, RO data at 1206 JST, were assimilated, respectively. In the experiments of 'RO+PWV' and 'RO+SWV', RO and PWV data, or RO and SWV data were assimilated simultaneously.

Figure 5 is the distributions of 3-hour rainfall from 15 JST to 18 JST predicted from the analyzed fields of these five experiments. When RO data were assimilated ('RO'), a rainfall region, of which 3-hour rainfall amount was over $10 \mathrm{~mm}$, was generated in northern Japan. Besides this rainfall region, more intense rainfalls appeared on the eastern side of northern Japan. This feature is also seen in 'CNTL'. Compared with the observed rainfall regions (Fig. 2a), the rainfall region in the northern Japan was more elongated in the north-south direction. When PWV or SWV data were assimilated ('PWV' or 'SWV'), the rainfall region in northern Japan was also reproduced. In contrast to the 'RO' case, the rainfall region was narrower than and similar to the observed one. However, the intensity was weaker than that of the 'RO' case. Besides the rainfall regions in northern Japan, rainfall regions in western Japan, which were not generated in 'RO', were reproduced, though their rainfall amount was too intense. This advantage of PWV and SWV data was yielded by their wider data coverage

When RO and PWV were assimilated ('RO+PWV'), the rainfall in northern Japan became stronger than that of 'RO' or 'PWV'. The rainfall regions in western Japan were also reproduced. The intense rainfall region on the eastern side of northern Japan, which was generated in 'RO', remained in 'RO+PWV'. When RO and SWV data were used as assimilation data ('RO+SWV'), the intensity of the rainfall in northern Japan was slightly intensified than that in 'SWV'. The rainfall on the eastern side of northern Japan became weaker than that in 'RO'.

Next, the difference of water vapor from the analyzed field in 'CNTL' is shown in Fig. 6. When PWV data were assimilated, water vapor in the rainfall region was increased and on the north of the rainfall region was decreased. These differences were consistent with the D-value distributions (Fig. 3b). In addition to these differences, water vapor east of northern Japan was decreased when SWV data were assimilated. When RO data were assimilated, water vapor below the height of $4 \mathrm{~km}$ was increased (Fig. 7). This range of height was consistent with the height where the observed RIs were larger than those of the first guess (Fig. 4). This vertical contrast in water vapor profile was higher than that in 'PWV' and 'SWV'. However, the decreased region of water vapor north of rainfall region was not reproduced by the assimilation of 'RO', because any information on the horizontal distribution was not given in 'RO'. As for the horizontal wind, a southeasterly wind at the eastern side of northern Japan (white circles in Fig. 6) was intensified by the assimilation of RO data. This difference of (a) Observation

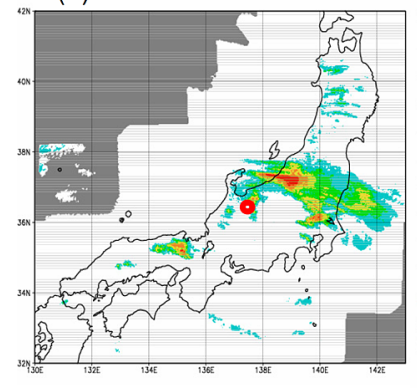

(b) CNTL

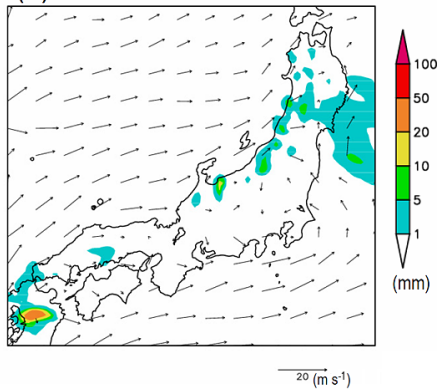

Fig. 2. (a) Three-hour rainfall from 15 JST to 18 JST 16 July 2004, which was observed by conventional radar and rain gauge data. Red circle indicates the tangent point of the lowest path. (b) Three-hour rainfall and horizontal wind near surface predicted from the analyzed fields of conventional data.
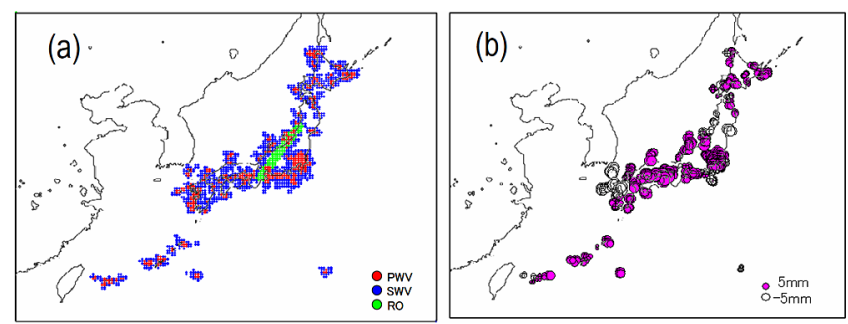

Fig. 3. (a) Grids distribution of which spatial interpolation weight was positive in assimilation of ground-based GPS data and RO data. (b) D-value of PWV between the observations and the first guess from 12 JST to 15 JST.

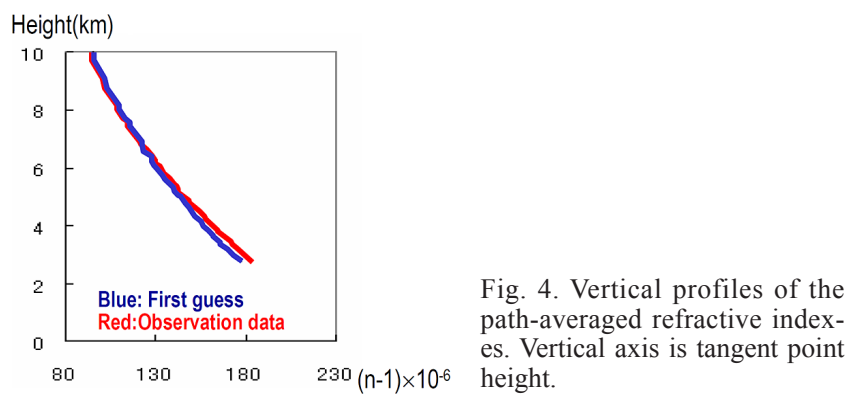

the horizontal wind reinforced the rainfall at the eastern side of northern Japan through the intensified convergence.

When ground-based GPS data and RO data were assimilated simultaneously, water vapor field was improved horizontally and vertically at the same time. For example, water vapor below the height of $4 \mathrm{~km}$ was increased in the rainfall region and on the southern side, and on the north of the rainfall region was decreased in ' $\mathbf{R O}+\mathbf{P W V}$ '. The southeasterly wind at the eastern side of northern Japan was also intensified in both cases. Because water vapor east of northern Japan was not decreased in 'RO+ PWV', the intense rainfall at the eastern side of northern Japan was produced by the intensified convergence. In contrast, the rainfall remained weak even under the intensified convergence in ' $\mathbf{R O}+\mathbf{S W V}$ ' because water vapor east of northern Japan was decreased.

Among the five experiments, the rainfall region in ' $\mathbf{R O}+\mathbf{S W V}$ ' is most similar to the observed one. When SWV data were assimilated instead of PWV in 'RO+PWV', water vapor on the north and east of the rainfall region was more decreased and the positive region of the D-value became more compact (Figs. 6 and 7). These comparisons show that SWV can modify the horizontal distribution of water vapor more effectually, even if the horizontal 

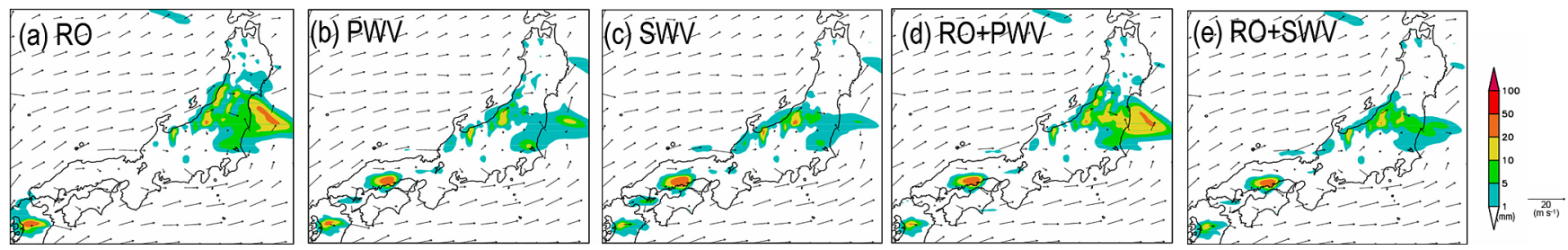

Fig. 5. Reproduced 3-hour rainfall of forecast time from 0 to 3 hours reproduced from the analyzed fields. Valid time is 15 to 18 JST 16 July 2004.
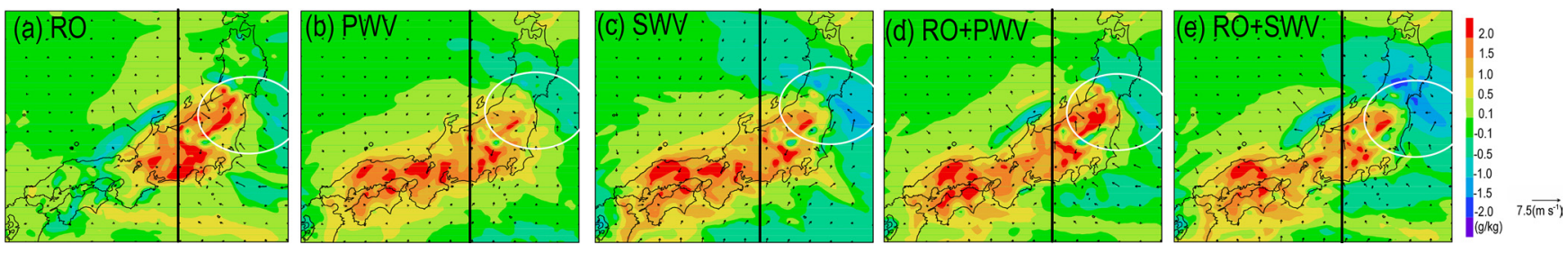

Fig. 6. Horizontal distributions of the difference of analyzed water vapor from 'CNTL' at the height of $21 \mathrm{~m}$. Black lines indicate the position of the vertical cross sections of Fig. 7.
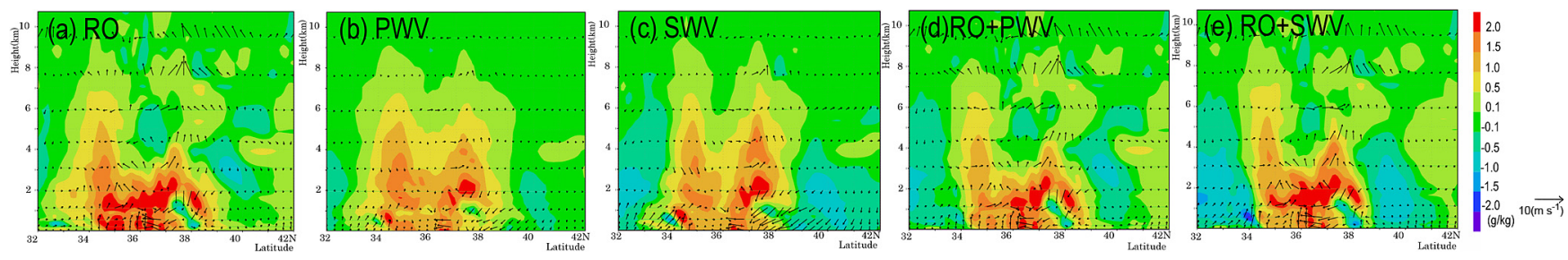

Fig. 7. Vertical distribution of the difference of analyzed water vapor from 'CNTL' at the latitude of $138 \mathrm{deg}$. Vectors represent horizontal wind direction and velocity.

grid of model was $20 \mathrm{~km}$. The rainfall region in ' $\mathbf{R O}+\mathbf{S W V}$ ', which is most similar to the observed one, suggests that the following two modifications seem to be important to reproduce the intense rainfall of this case; (1) the high contrast of water vapor reproduced by SWV data, and (2) the low-level humid air in the rainfall region in east Japan that was produced by $\mathrm{RO}$ data.

\section{Summary}

Results of this study are summarized as follows;

(1) When SWV, PWV and RO data were assimilated, the rainfall in northern Japan was reproduced.

(2) When ground-based GPS data were assimilated, the contrast of water vapor distribution in the horizontal direction became larger, and the rainfall distribution became closer to the observed one. When RO data were used, the low-level water vapor in the rainfall region was increased so that rainfall intensity was greatly reinforced.

(3) When ground-based GPS data and RO data were assimilated simultaneously, water vapor fields in the horizontal and vertical sections were improved.

(4) SWV modified the water vapor distribution more effectively than PWV did even if the grid interval was $20 \mathrm{~km}$. Simultaneous assimilation of SWV and RO produced the rainfall system closest to the observed one, because SWV made the contrast of water vapor along the northern side of the rainfall region and $\mathrm{RO}$ increased the low-level water vapor.

\section{Acknowledgements}

CHAMP data and GPS data were provided from the GFZ and GSI. The Meso 4D-Var system was developed by the Numerical
Prediction Division (NPD) of JMA. Authors would like to express their appreciation to GFZ, GSI and NPD of JMA. Authors' gratitude extends to anonymous referees who provided useful comments.

\section{References}

Koh, T. Y., and C. K. Teo, 2009: Towards a mesoscale observation network in Southeast Asia. Bull. Am. Meteor. Soc., 90, 481-488.

Koizumi, K., Y. Ishikawa, and T. Tsuyuki, 2005: Assimilation of precipitation data to the JMA mesoscale model with a fourdimensional variational method and its impact on precipitation forecasts. SOLA, 1, 45-48.

Seko, H., T. Kawabata, T. Tsuyuki, H. Nakamura, K. Koizumi, and T. Iwabuchi, 2004: Impacts of GPS derived water vapor and radial wind measured by Doppler Radar on numerical prediction of precipitation. J. Meteor. Soc. Japan, 82, 473-489.

Seko, H., Y. Shoji, M. Kunii, and Y. Aoyama, 2009: Impact of the CHAMP Occultation Data on the Rainfall Forecast, Data Assimilation for Atmospheric, Oceanic and Hydrologic Applications, 197-217.

Shoji, Y., H. Nakamura, T. Iwabuchi, K. Aonashi, H. Seko, K. Mishima, A. Itagaki, R. Ichikawa, and R. Ohtani, 2004: Tsukuba GPS Dense Net Campaign Observation: Improvement in GPS analysis of slant path delay by stacking one-way postfit phase residuals. J. Meteor. Soc. Japan, 82, 301-314.

White, B. G., J. Paegle, W. J. Steenburgh, J. D. Horel, R. T. Swanson, L. K. Cook, D. J. Onton, and J. G. Miles, 1999: Shortterm forecast validation of six models. Wea. Forecasting, 14, 84-108.

Yoden, S., 2007: Atmospheric predictability. J. Meteor. Soc. Japan, 85B, $77-102$.

Manuscript received 10 February 2010, accepted 11 May 2010

SOLA: http://www.jstage.jst.go.jp/browse/solal 